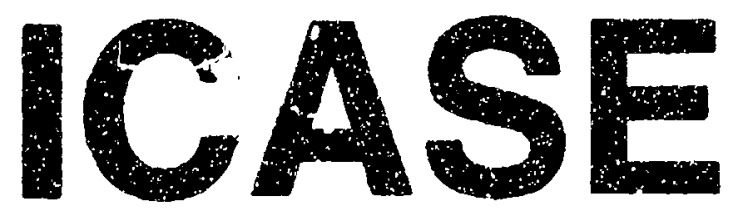

\title{
ANALXSIS OF THE HESSIAN FOR AERODYNAMIC OPTIMIZATION: INVISCID FLOW
}

\section{Eyal Arian \\ Shlomo Ta'asan}

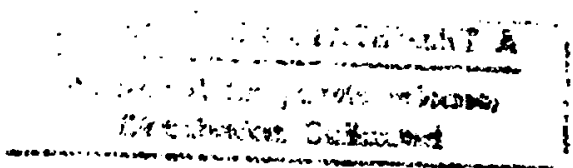

NASA Contract No. NASI-19480

April 1996

Institute for Computer Applications in Science and Engineering

NASA Langley Research Center

Hampton, VA 23681-0001

Operated by Universities Space Research Association

NASA

National Aeronautics and Space Administration

Langley Research Center

Hampton, Virginia 23681-0001

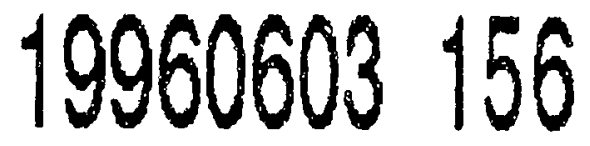

DITC QOALTTY DIEPECTED I 

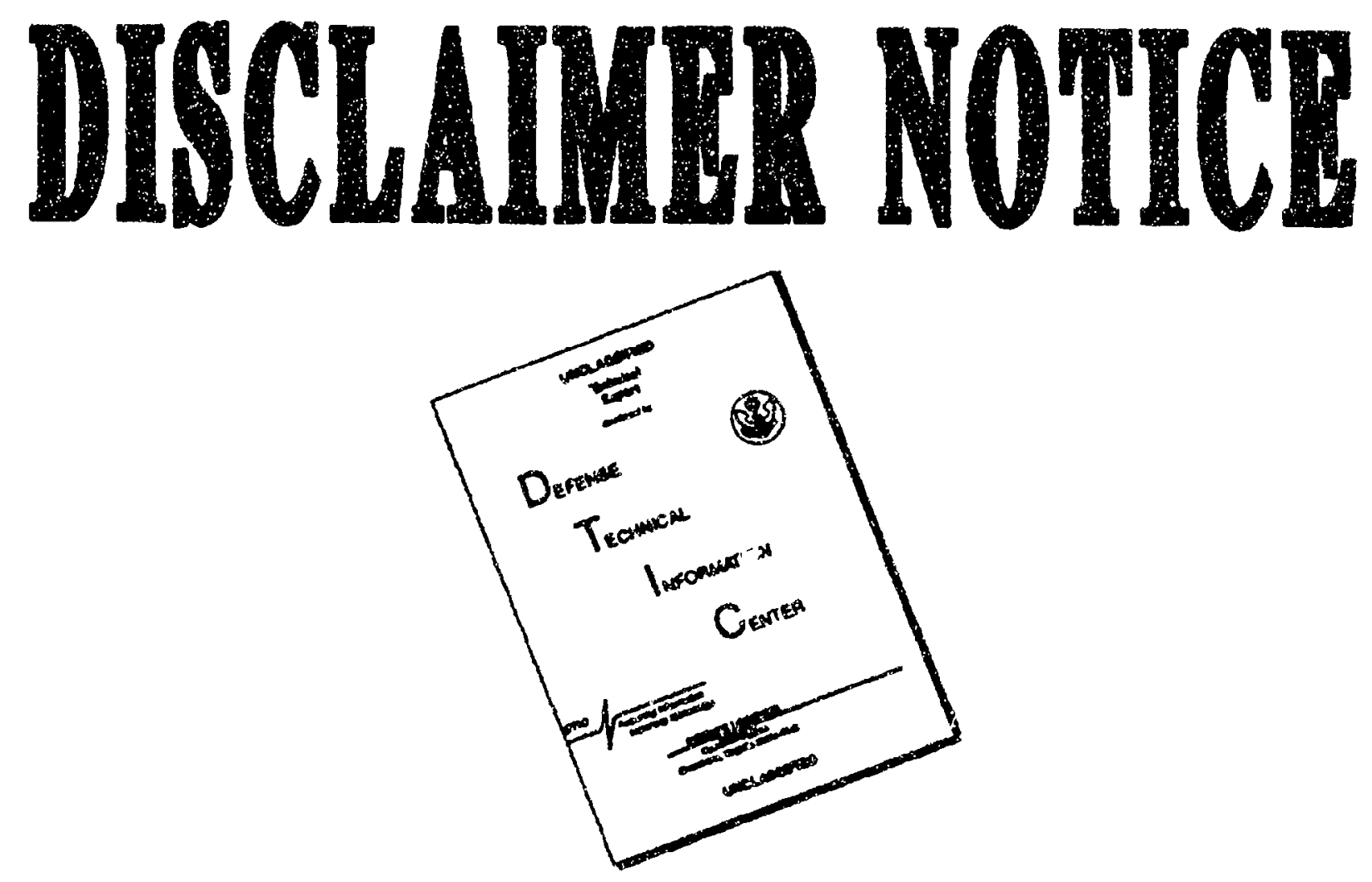

THIS DOCUMENT IS BEST

QUALITY AVAILABLE. THE COPY

FURNISHED TO DTIC CONTAINED

A SIGNIFICANT NUMBER OF

PAGES WHICH DO NOT

REPRODUCE LEGIBLY. 


\title{
Analysis of the Hessian for Aerodynamic Optimization: Inviscid Flow *
}

\author{
Eyal Arian \\ Institute for Cornputer Applications in Science and Engineering \\ Mail Stop 132C, NASA Langley Research Center \\ Hampton, VA 23681-0001, USA
}

\author{
Shlomo Ta'asan \\ Department of Mathematics \\ Carnegie-Mellon University \\ Pittsburgi. PA 15213, USA
}

\begin{abstract}
In this paper we analyze inviscid aerodynamic shape optimization problems governed by the full potential and the Euler equations in two and three dimensions. The analysis indicates that minimization of pressure dependent cost functions results in Hessians whose eigenvalue distributions are identical for the full potential and the Euler equations. However, the optimization problems in two and three dimensions are inherently different. While the iwo dimensional optimization problems are well-posed. the three dimensional ones a re ill-posed. Oscillations in the shape up to the smallest scale allowed by the design space can develop in the direction perpendicular to the flow, implying that a regularization is required. A natural choice of such a regularization is derived. The analysis also gives an estimate of the Hessian's conditicn number which implies that the problems at hand are ill-conditioned. Infinite dimensional approximations for the Hessians are constructed and preconditioners for gradient based methods are derived from these approximate Hessians.
\end{abstract}

-This research wasi supported by the National Aeronautics and Space Administration under NASA Contract No. NASl-18480 while the authors wese in residence at the Institute for Computer Applications in Science and Engineering (ICASE), Mail Stop 132C, NASA Langle: Research Cenler. Hamptors. VA 236810001 . 


\section{Introduction}

In recent years there has been a growing interest in solving optimization problems governed by the Euler and the Navier Stokes equations $[1 ;-99 j$. The new interest in this classical field $[10,11]$ is due to the increase in computer's speed and improvements in algorithms for the numerical solution of the flow equations.

The problem of designing a three-dimensional wing requires solving an optimization problem with many design paramelers. Such a problem may be computationally difficult depending on the cost function's level curves in the vicinity of the minimum. A measure for the level of difficulyy is the condition number of the Hessian. The eigenvalues of the Hessian (which is a symmetric operator) are the curvatures of the cost function in the principal directions. A large deviation in the eigenvalues means that the cost function has level curves which are thin ellipses. This is well known in the optimization literature to cause slowness of convergence toward the minimum for gradient-based methods $[12$;.

Aerodynarnic optimization problems are ill-conditioned as noted in [6.9, and as will be shown in this paper. Therefore gradient descent methods will be ext.remely inefficient especially when the number of design variables is large. A standard method to overcome this difficulty is the Newton method where the Hessian is computed explicitly [12]. The Newton search direction is the gradient multiplied by the Hessian's inverse, a computation which is impractical in aerodynamic optimization problems since it involves numerous solutions of the flow PDEs. On the other hand ssing low rank quasi-Newton methods, such as BFGS [3. 4]. wiil result in a deteriorace con erzence as the number of design variables increases. Thus, a new rnethod is required.

Another difficulty in inviscid aerodynan. optimization problems is the ill-posedness of three dimensional problems which shows up as small scale oscillations in the shape in the direction perpendicular to the flow. Such oscillations were observed in applications as reported in [9]. One way to avoid these oscillations is to apply smooth finite dimensional representation of the shape in the spanwise direction. Another approach is regularization by introduction of a penalty to the cost function for ascillations in that direction. The need for penalizing the cost function in order to rernove oscillations was observed by [3] However, in that case the oscillations were a result of the discretization and had no differential counterpart. Penalization was used also in two dimensions where the differential optimization problem is well-posed.

In this paper we develop a new approach to approsimate the Hessian and its inverse for optimization problems governed by PDEs. Hessian symbols were previously computed far smoothing predictions in the development of multigrid one-shot methods [13]-[16]. Ilere, a similar analysis is applied to inviscid flow problems including the full potential and Euler equations in two and in three space dimensions. In Sec.2 the optimization problem is defined together with its small disturbance approximation. The necessary conditions for a minimum and their relation with the Hessian are discussed also in the finite dimensional design space. In Sec. 3 local mode analysis is presented to approximate the Hessian's symbol. The dnalysis is local and involves freezing the coefficients to obtain a problem in half space with constant coeficients, where Fourier techniques are employed. In Sec. 4 the analysis is applied to an optimal shape design problem governed by the full potential equation. In Sec. 5 the analysis is applied to the Euler equations. and a symbol is obtained identical to the full potential 
case. In Sec. 6 the Hessian's symbol is analyzed and conclusions are made concerning the ill. conditioning and ill-posedness of the problems at hand. In Sec. 7 regularization is discussed to avoid the ill-posedness which exists in three dimensions in the spanwise direction. In Sec. 8 preconditioners are developed for the small disturbance and the optimal shape problems in subsonic and supersonic flow. Finally in Sec. 9 discussion and concluding remarks are made.

\section{The Optimization Problem}

A typical inviscid aerodynamic optimal shape design problem aims at finding the shape of a surface, e.g. airfoil or wing, such that the resulting pressure distribution on that surface will minimize the least squares distance from a prescribed pressure distribution. Let $\Omega$ be a domain in $\mathbb{R}^{d}$ and $\Gamma(x)$ a parametric representation of the part of the boundary $\partial \Omega$ to be designed. The optimal shape problem is to compure the boundary position, $\Gamma$. that minimizes a cost function defined on $\Gamma . e . g$.,

$$
\min _{\Gamma} \int_{\Gamma} f(U) d \sigma
$$

where $f$ is a prescribed function and $U$ is the solution of a PDE defined on $\Omega$,

$$
\mathcal{L}(\Gamma, U)=0 .
$$

\subsection{The Small Disturbance Approximation}

For the analysis of the Hessian it is enough to consider small perturbations of the boundary $\Gamma$. In order to further simplify the derivation we consider a localization of the problem in a vicinity of a boundary point and study the resulting half space problem. Let us introduce the following notation

$$
\begin{aligned}
\mathbb{R}_{+}^{n} & \equiv\left\{\left(\vec{x}, x_{n}\right): \vec{x} \in \mathbb{R}^{n-1}, 0<x_{n}\right\} \\
\partial \mathbb{R}_{+}^{n} & \equiv\left\{\left(\vec{x} . x_{n}\right): \vec{x} \in \mathbb{R}^{n-1}, x_{n}=0\right\} .
\end{aligned}
$$

We consider perturbations of the form

$$
\begin{array}{r}
\Gamma=\Gamma^{*}+\varepsilon \tilde{\alpha} \bar{n} \\
I=l^{*}+\varepsilon \hat{U}+O\left(\varepsilon^{2}\right)
\end{array}
$$

where $\Gamma^{*}$ and $l^{*}$ are the optimal boundary shape and state solution respectively; $\bar{n}$ is the outward normal and $\varepsilon$ is a sufficiently small positive number. The resulting optimal control problem is obtained using a Taylor expansion in $c$ and is discussed in more details in the next sections. The new control problem, for the control variable $\alpha$, is defined in half space and is referred as the "small disturbance minimization problem", namely,

$$
\min _{\ddot{\alpha}} F(\ddot{\alpha}, \tilde{l})
$$

subject to the following state equation:

$$
\begin{array}{rr}
\mathrm{L} \dot{l}=0 & \text { on } \mathbb{R}_{+}^{3} \\
\mathrm{~B} \dot{L}^{i}=\mathrm{C} \dot{\alpha} & \text { on } \partial R_{+}^{3}
\end{array}
$$


and additional homogeneous conditions on the solution at infinity. For this control problem the minimum is attained for $\ddot{\alpha}=0$.

The necessary conditions for a mininum of the small disturbance problem are given by the state equation Eq.(2.6-2.i) and two additional equations: design and costate. The design equation is a PDE defined on the boundary $\partial \mathbb{R}_{+}^{3}$ and is denoted by;

$$
\mathcal{A}(\hat{U}, \Lambda, \dot{\alpha})=0 \quad \text { on } \partial \mathbb{R}_{+}^{3}
$$

where $\dot{l}$ is the solution of the state equation $(2.6-2.7)$ and $\Lambda$ is the solution of the costateequation. i.e.,

$$
\begin{array}{rr}
\tilde{\mathrm{L}} \Lambda=0 & \text { on } \mathbb{R}_{+}^{3} \\
\tilde{\mathbf{G}} \Lambda=\tilde{\mathbf{C}} l^{i} & \text { on } \partial \mathbb{R}_{+}^{3}
\end{array}
$$

and additional bomogeneous conditions at infinity.

We assume that the perturbation $\tilde{a}$ is composed of high frequencies and compute the resulting solutions, $\tilde{U}$ and $\Lambda$, in the vicinity of some arbitrary point on the boundary $\partial R_{+}^{3}$. The solution there is approximated by a constant coefficient problem defined in half space where Fourier analysi can be applied.

It can be shown that for feasible solutions of the state and costate equations. $\bar{U}=\bar{l}(\dot{\alpha})$ and $\Lambda=\Lambda(\tilde{C}(\tilde{\alpha}) ;$. the design equation residuals are equal to the gradient of the cost function with respect to the design variables:

$$
g(\tilde{\alpha}, \check{l}(\tilde{\alpha}))=\mathcal{A}(\tilde{U}(\tilde{\alpha}), A(\tilde{l}(\tilde{\alpha})), \tilde{\alpha}) .
$$

In the vicins ine minimum the following relation holds

$$
g(\dot{\alpha} \cdot \tilde{U}(\dot{\alpha}))=g(0,0)+H \dot{\alpha}+k .0 . t
$$

where ve dencte by $H$ the Hessian, i.e.:

$$
H=\nabla_{\dot{\alpha}} g(0,0)
$$

A Taylor expansion. of the right hand side in (2.11) and comparison with (2.12) results in

$$
H=\mathcal{A}_{\tilde{U}} \tilde{l}_{\dot{\alpha}}+\mathcal{A}_{\Lambda} \Lambda_{i} \tilde{l}_{\dot{\delta}}+\mathcal{A}_{\dot{\delta}}
$$

The dimension of the Hessian. $H$. is determined by the dimension of the design variable. $\tilde{\alpha}$. If $\dot{\alpha}$ belong to a finite dimensional space of dimension $N$ then the gradient is a vector of size $N$ and the Hessian is a $N \times N$ matrix. If $\dot{\alpha}$ belongs to an infinite dimensional space (e.g., some function space) then the gradient is an element in an infinite dimensional space and the Hessian is an infinite dimensional operator. The Newton step, $\dot{\alpha}$, satisfies

$$
H \tilde{\alpha}=-g
$$

where $g$ is the gradient at the given iteration. 


\subsection{Finite Dimensional Design Space}

In applications it is a common practice to restrict the design space to a finite dimensional subspace where the shape $\Gamma$. or in the small disturbance model $\tilde{a}$, is given as

$$
\dot{a}(s)=\sum_{j=1}^{N} \dot{\alpha}_{j} f_{j}(s) .
$$

where $\lambda$ is the dimension of the design space. $\alpha_{j}$ are the design variables and $f_{j}$ are fixed basis functions. The gradient for the finite dimensional case, $\vec{g}=\left(g_{1}, \cdots, g_{v}\right)$, is obtained by a projection of the infinite dimensional one onto on the finite dimensional space, i.e.,

$$
J \vec{g}=\vec{b}
$$

where $J$ is a matrix defined by

$$
J_{i j}=\left(f_{i}(s), f_{j}(s)\right)_{L^{2}} \quad i_{i} j=1, \cdots, N \text {, }
$$

and the right hand side, $\vec{b}$, is a vector whose elements are given by

$$
b_{k}(s)=\left(g(s), f_{k}(s)\right)_{L^{2}} \quad k=1, \cdots, \lambda .
$$

Let $h_{j k}$ be an element in the matrix representing the Hessian using the basis functions $f_{j}$ :

$$
h_{j k}=\left(H f_{j} \cdot f_{k}\right)_{L^{2}} \text {. }
$$

A Newton step for minimizing the cost function consists of moring in the direction $\overrightarrow{\tilde{\alpha}}=$ $\left(\tilde{\alpha}_{1}, \cdots, \dot{\alpha}_{v}\right)$ given by:

$$
h \overrightarrow{\tilde{a}}=-\vec{b} \text {. }
$$

Sote that this equation is obtained by projecting the general equation (2.14) onto a finite dimensional space spanned by $\left\{f_{j}\right\}_{j=1}^{N}$.

\section{Local Mode Analysis}

A local analysis of the operator $H$ is done next. The Fourier analysis consists of analyzing the solution of the following system of equations in half space:

$$
\begin{aligned}
& L \tilde{\bar{l}}=\overrightarrow{0} \quad \text { on } \mathbb{R}_{+}^{n} \\
& \mathrm{~B} \dot{\vec{l}}=\mathrm{C} \dot{\alpha} \quad \text { on } \partial R_{\downarrow}^{n} \\
& \mathbf{L} \vec{i}=\overrightarrow{0} \quad \text { on } \mathbb{R}_{+}^{n} \\
& \mathbf{B} \bar{\Lambda}=\bar{C} \overline{\vec{l}} \quad \text { on } \partial R_{+}^{n} \\
& \mathcal{A}(\dot{\bar{L}}, \vec{\Lambda} . \tilde{\alpha})=0 \quad \text { on } \partial R_{+}^{n} \text {, }
\end{aligned}
$$


where $u$ e replaced $\dot{U}$ by $\overrightarrow{\vec{l}}$ to account for cases in which $\mathbf{L}$ stands for a system of PDEs.

The computation of the symbol of the Hessian. (2.13), near the minimum is done by considering a perturbation in the design variable of the form

$$
\tilde{\alpha}(\vec{x})=\hat{\alpha}(\vec{k}) e^{i \vec{k} \cdot \vec{r}}
$$

and calculating the corresponding term $H \tilde{\alpha}$. The small disturbance solution $\dot{\bar{U}}$ can be represented as

$$
\tilde{\vec{U}}\left\{\vec{x} . x_{n}\right\}=\sum_{j=1}^{q} \beta_{j}(\vec{k}) \hat{\vec{V}}_{j}(\vec{k}) e^{i \vec{k} \cdot \vec{x}} \epsilon^{i k_{n}^{3} x_{n}}
$$

where $q$ equals the number of boundary conditions in (3.2). Each of the terms in the expression for $\overrightarrow{\vec{b}}$ satisfies the equation

$$
\mathbf{L} \hat{\vec{V}} ;(\vec{k}) \epsilon^{i \vec{k} \cdot \vec{x}} \epsilon^{i k_{n}^{\prime} x_{n}}=\overrightarrow{0} .
$$

which implies

$$
\dot{L}\left(\vec{k}, k_{n}^{j}\right) \hat{\vec{V}}_{3}(\vec{k})=\overrightarrow{0}
$$

where $\dot{L}\left(\vec{k}, k_{n}^{\prime}\right)$ is the symbol of L. Moreover, Eq. $(3.9)$ implies that for $j=1 \cdots q$,

$$
\operatorname{det} \hat{L}\left(\vec{k}, k_{n}^{j}\right)=0
$$

and that $\dot{\vec{V}}_{j}\left(\vec{k}_{i}\right)$ is an eigenvector of $\dot{L}\left(\vec{k}, k_{r}^{j}\right)$ with a zero eigenvalue. Substituting the expres sion for $\overrightarrow{\vec{i}}$ into the boundary condition (3.2) results in the following equation.

$$
\sum_{j=1}^{q} \beta_{j}(\vec{k}) \hat{B}\left(\vec{k}, k_{n}^{j}\right) \hat{\vec{V}}_{j}(\vec{k})=\hat{C}(\vec{k}) \hat{\alpha}(\vec{k})
$$

where $\hat{B}\left(\vec{k} . k_{n}^{j}\right)$ is the symbol of $\mathbf{B}$. and $\hat{C}(\vec{k})$ is the symbol of $\mathbf{C}$. Introducing the matrix

$$
\hat{H}(\vec{k})=\left(\hat{B}\left(\vec{k}, k_{n}^{1}\right) \hat{\vec{V}}_{1}(\vec{k}), \ldots, \dot{B}\left(\vec{k}, k_{n}^{o}\right) \hat{\vec{V}}_{g}(\vec{k})\right)
$$

and the vector

$$
\vec{\beta}(\vec{k})=\left(\beta_{1}^{3}(\vec{k}), \ldots, \beta_{4}(\vec{k})\right)
$$

Eq.(3.11) can be: written as

$$
\hat{W}(\vec{k}) \dot{\vec{\beta}}(\vec{k})=\dot{C}(\vec{k}) \dot{\alpha}(\vec{k})
$$

or, equivalently, as

$$
\hat{\vec{U}}(\vec{k})=\hat{V}(\vec{k}) \hat{l}^{-1}(\vec{k}) \hat{C}(\vec{k}) \hat{\alpha}(\vec{k})
$$

where $\hat{\vec{l}}(\vec{k})$ is defired by

$$
\dot{\vec{l}}(\vec{x} \cdot 0)=\hat{\vec{V}}(\vec{k}) \dot{\vec{B}}(\vec{k}) e^{i \vec{k} \cdot z} \equiv \hat{\vec{U}}(\vec{k}) e^{i \vec{k} \cdot \vec{z}}
$$


and where $\dot{V}(\bar{k})$ is the matrix $\hat{V}=\left(\hat{\vec{V}}_{1}(\vec{k}), \ldots \dot{\vec{V}}_{q}(\vec{k})\right)$.

The adjoint equations are treated in a similar way. The solution $\Lambda$ is represented by

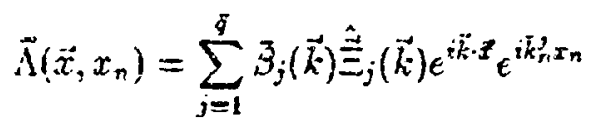

where $q+\bar{q}=N$ and $N$ is the degree of the polynomial in (3.10). Let $\bar{k}_{n}^{j}$ be the roots of

$$
\operatorname{det} \hat{\bar{L}}\left(\vec{k}, \bar{k}_{n}^{j}\right)=0
$$

and $\vec{\Xi}_{j}$ the eigenvectors of $\vec{L}\left(\vec{k}, \vec{k}_{n}^{j}\right)$ corresponding to a zero eigenvalue. The costate boundary condition (3.1) implies

$$
\sum_{i=1}^{\Phi} \bar{B}_{j}(\vec{k}) \hat{\bar{B}}\left(\bar{k}_{i}, \bar{k}_{n}\right) \hat{\bar{\Xi}}_{j}(\vec{k})=\hat{C}(\vec{k}) \hat{\vec{C}}(\vec{k})
$$

Introducing the matrix

$$
\hat{\bar{W}}(\vec{k})=\left(\hat{\bar{B}}\left(\vec{k}, \bar{k}_{n}^{1}\right) \hat{\vec{\Xi}}_{1}(\vec{k}), \ldots, \hat{\bar{B}}\left(\vec{k} \cdot \bar{k}_{n}^{\bar{j}}\right) \hat{\hat{\Xi}}(\vec{k})\right)
$$

and the vector

$$
\overrightarrow{\bar{\beta}}(\vec{k})=\left(\bar{\beta}_{1}(\vec{k}) \ldots, \ddot{\beta}_{q}(\vec{k})\right)
$$

Eq.(3.16) can be uritten as

$$
\hat{\bar{W}}(\tilde{k}) \hat{\overline{\hat{U}}}(\vec{k})=\hat{\vec{C}}(\ddot{k}) \hat{\vec{U}}(\vec{k})
$$

or. equivalently. as

$$
\dot{\vec{\Lambda}}(\vec{k})=\dot{\Xi}(\vec{k}) \hat{W}^{-1}(\vec{k}) \hat{C}(\vec{k}) \hat{\vec{U}}(\vec{k})
$$

where $\hat{\Xi}(\vec{k})$ is the matrix $\hat{\Xi}(\vec{k})=\left(\dot{\vec{\Xi}}_{1}(\vec{k}), \ldots, \hat{\doteq}_{\gamma}(\vec{k})\right)$.

Substitution of (3.13) and (3.18) in the symbol of the Hessian's expression (2.13) results in the following formula for the Hessian's symbol:

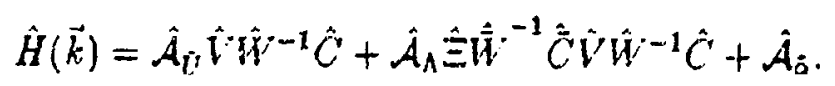

\section{The Full Potential Equation}

In this section we apply the ideas discussed above to minimization problems governed by the full potential equation. We consider the problem of optimizing the shape of an aerody namics 
configuration so that the shape minimizes the deviation of the pressure from a target pressure distribution. namely.

$$
\min _{\Gamma} F(\Gamma, p)=\int_{\Gamma}\left(p-p^{*}\right)^{2} d \sigma
$$

subject to

$$
\begin{aligned}
\mathcal{L}(\Gamma, \phi) \equiv \operatorname{div}(\rho \nabla \phi) & =0 & & \text { on } \Omega \\
\phi_{n} & =0 & & \text { on } \Gamma
\end{aligned}
$$

and an additional boundary condition at the far-field (the notation $\dot{\phi}_{n}$ stands for the outer normal derivative of $\phi$ on the boundary).

The density. $\rho$. is given by the isentropic density law [1 $T$ :

$$
\frac{\rho}{\rho_{\infty}}=\left\{1-\frac{(\nabla \dot{\phi})^{2}}{2 H_{\infty}}\right\}^{\frac{1}{x-3}}
$$

with $\gamma$ the specific heat ratio and $\rho_{\infty}$ and $H_{\infty}$ the values of the density and total enthalpy at infinity.

The pressure. $p$, is related to the density $\rho$ and the speed of sound, $c$, by

$$
p=\frac{\rho c^{2}}{\gamma}
$$

where for perfect gases the speed of sound is related to the potential through the relation

$$
c^{2}=(\gamma-1)^{i} H_{x}-\frac{(\nabla \phi)^{2}}{2}
$$

The Mach number $H$ is given by

$$
M^{2}=\frac{1 \nabla_{\phi}^{\prime 2}}{c^{2}}
$$

\subsection{The Small Disturbance Minimization Problem}

The derivation of the small disturbance minimization problem follows the argument and notation of Sec.2.1. We perform localization and set the local coordinate system on the boundary such that the flow is in the $x$-direction $\left(\nabla \phi=\left(\phi_{x}, 0,0\right)\right)$. On the perturbed boundary a Taylor expansion gives [1]

$$
p^{\text {pertursed }}=p+\tilde{p}+\tilde{\alpha} p_{n}+\text { h.o.t. }
$$

and

$$
d \sigma^{\text {perturbed }}=\left(1-\frac{\dot{\alpha}}{R}\right) d \sigma+\text { h.o.t. }
$$

where $\frac{1}{R}=\frac{1}{R} \cdot+\frac{1}{R_{2}}$ and $R_{1}, R_{2}$ are the principal curvatures.

The small disturbance minimization problem is given by

$$
\min _{\dot{\alpha}} F(\tilde{\alpha}, \dot{l}) \equiv \int_{\partial R_{+}^{3}}\left(\left(p-y^{*}\right)+\tilde{p}(\dot{\phi})+\dot{\alpha} p_{n}\right)^{2} d x d y-\int_{\partial R_{+}^{*}} \frac{\dot{\alpha}}{R}\left(p-p^{*}\right)^{2} d x d y .
$$


subject to

$$
\left(1-M^{2}\right) \dot{\phi}_{x z}-\dot{\phi}_{y y}+\dot{\phi}_{z z}=0 \text { on } \mathbb{R}_{. .}^{3} \text {. }
$$

The small disturbance boundary condition is giver by

$$
-\dot{\phi}_{z}-\dot{\phi}_{x} \dot{\alpha}_{I}+\phi_{: z} \dot{\alpha}=0
$$

where the unperturbed flow is in "he $x$-direction. Since we are analyzing the Hessian for the high frequencies it is enough to consider

$$
-\dot{\phi}_{z}=\dot{\phi}_{x} \hat{\alpha}_{x}
$$

The relation between $\dot{p}$ and $\dot{\phi}$ in the cost function $(4.7)$ is obtained from the relations (4.4$4.6)$. We obtain that the relevant cost function in terms of $\dot{\phi}$ to be minimized is

$$
F\left(\dot{\alpha}_{0} \dot{\phi}\right)=\int_{\partial R_{+}^{2}}\left(\left(p-p^{*}\right)-\rho \dot{\phi}_{x} \tilde{\phi}_{x}\right)^{2} d x d y-\int_{\partial R_{+}^{3}} \tilde{\alpha}\left[\frac{1}{R}\left(p-p^{*}\right)^{2}-2 p_{n}\left(p-p^{*}\right)\right] d x d y .
$$

\subsection{The Adjoint Equations}

By standard variational calculus it can be shown that the gradient of the cost function is given by

$$
g=\phi_{x} \lambda_{x}-\frac{\left(p-p^{*}\right)^{2}}{R}+2 p_{n}\left(p-p^{*}\right)
$$

where $\lambda$ is the solution of

$$
\left(1-M^{2}\right) \lambda_{x x}+\lambda_{y y}+\lambda_{z z}=0 \quad \text { on } \mathbb{R}_{\div}^{3}
$$

with the wall boundary condition

$$
-\lambda_{2}-2\left[\rho \dot{\phi}_{i r}\left(\left(p-p^{*}\right)-\rho \dot{\phi}_{x} \dot{\phi}_{x}\right)\right]_{x}=0 \quad \text { on } \partial R_{+}^{3}
$$

and $\tilde{\phi}$ is the solution of (4.8) with the boundary condition (4.10). We also require that the solution is bounded for the subsonic case $(M<1)$ and that in the supersonic case $(M>1)$ no waves propagate in the direction of $-\nabla \delta\left(x_{0}\right)$, i.e., not propagating in the negative $x$ direction. This requirement is done so that the solution of the small disturbance problem will be consistent with the far-field boundary conditions of the unperturbed problem.

\subsection{Local Mode Analysis}

He now go through the analysis in Sec.3 in order to compute the symbol $\hat{H}(\vec{k})$ (see Eq.3.19) using the fuil potential state equations. Follcwing a perturbation $\tilde{\alpha}$

$$
\tilde{\alpha}(\vec{x})=\hat{\alpha}(\vec{k}) e^{i \vec{k} \cdot \vec{x}}
$$

the small disturbance solution, $\dot{\dot{o}}$. can be represented as

$$
\dot{\phi}\left(\overrightarrow{x^{\prime}}\right)=\dot{\phi}(\vec{k}) e^{i \vec{k} \cdot E^{\prime k_{3} z}},
$$


and. similarly; the adjoint variable,

$$
\lambda(\vec{x})=\hat{\lambda}(\vec{k}) e^{i \vec{k} \cdot z} \epsilon^{i k_{3} z}
$$

Substitution of $(4.15)$ in (4.8) and of (4.16) in (4.13) results in the following relations for $k_{3}$ and for $\bar{k}_{3}$ :

$$
\begin{gathered}
\left(1-M^{2}\right) k_{1}^{2}+k_{2}^{2}+k_{3}^{2}=0 \\
\left(1-M^{2}\right) k_{1}^{2}+k_{2}^{2}+\bar{k}_{3}^{2}=0
\end{gathered}
$$

These are the analogs of equations (3.10) and (3.15) respectively.

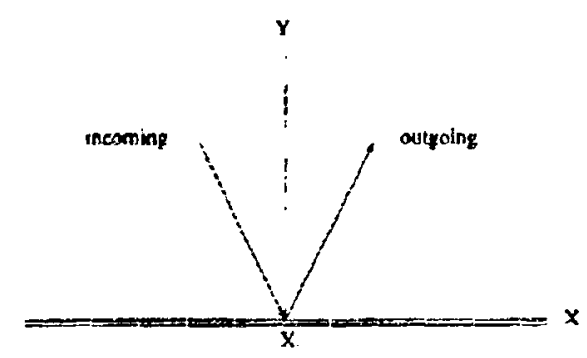

Figure 1: The supersonic flow can be decomposed into two waves: incoming and outgoing the plane $\partial R_{+}^{3}$.

The choice of sign for $k_{3}$ and $k_{3}$ is done as follows. Since the half space problem is related to localization of the original problem around some point $x_{0} \in \Gamma$, the solutions that we construct in half space should be compatible with the far field boundary conditions of the original problem. In the supersonic regime the solution can be decomposed into two waves: incoming and outgoing of the plane $\partial R_{+}^{3}$ (see Fig.1). In terms of the local coordinate system, the incoming characteristic has a component. in the negative $y$-direction while the outgoing has a component in the positive $y$-direction (both propagate in the positive $x$-direction). Since perturbations of the shape $\Gamma$ can not change the far field inflow data; the change in the inconing characteristic should vanish. Thus, the part of $\bar{\phi}$ which propagates to the negative $x$-direction is set to zero. This implies that $k_{3}$ is of opposite sign to that of $k_{1}$ when $k_{3}$ is real valued (i.e. supersonic flow). The adjoint variable has characteristics in the opposite direction and therefore we require that the part of the solution for $\lambda$ which propagates to the positive $x$-direction should be set to zero. As a result the sign of $\bar{k}_{3}$ is the opposite of that of $k_{3}$ if they are real valued. In the subsonic regime $k_{3}$ and $\bar{k}_{3}$ are imaginary therefore the proper sign is positive for both so that exponentially decaying solutions are obtained. Therefore $k_{3} k_{3}=-\left|k_{3}\right|^{2}$ for both subsonic and supersonic flow.

Frorn the boundary condition $(4.10)$ we obtain a relation between $\hat{\alpha}(\vec{k})$ and $\hat{\phi}(\vec{k})$, (as in (3.13))

$$
\dot{\phi}(\vec{k})=-\phi_{x} \frac{k_{1}}{k_{3}} \hat{\alpha}(\vec{k})
$$


and from the boundary condition (4.14) (as in (3.18))

$$
\hat{\lambda}(\vec{k})=2 i \rho^{2} \dot{\phi}_{x}^{2} \frac{k_{1}^{2}}{k_{3}} \dot{o}(\vec{k})
$$

Hence. the gradient (4.12) in Fourier space is

$$
\hat{g}(\vec{k})=\phi_{x} i k_{1} \hat{\lambda}(\vec{k})+\text { l.o.t. }
$$

Substitution of $(4.19)$ in $(4.20)$ and of $(4.20)$ in (4.21) results in the relation

$$
\hat{g}(\ddot{k})=2 \rho^{2} \dot{\phi}_{x}^{4} \frac{k_{1}^{4}}{k_{3} k_{3}} \hat{\alpha}=-2 \rho^{2} \phi_{x}^{4} \frac{k_{1}^{4}}{\left|k_{3}\right|^{2}} \hat{\alpha}
$$

and thus by relation (4.17) we obtain the following formula for the symbol of the Hessian:

$$
\hat{H}(\vec{k})=2 \rho^{2} \dot{\phi}_{x}^{4} \frac{k_{1}^{4}}{\left(1-M^{2}\right) k_{1}^{2}+k_{2}^{2} !} .
$$

\section{The Euler Equations}

As in the full potential case we consider the problem of optimizing the shape of an aerodynamic configuration. subject to the Euler equations, such that the optimal shape minimizes the deviation of the pressure from a target pressure distribution (4.1). We perform the analysis away from shocks so that it can be done using a non-conservative formulation. The Euler equations in quasi-linear non-conservative form are given by

$$
\mathcal{L}(\Gamma, \widetilde{L}) \equiv\left(\begin{array}{ccccc}
Q & \rho \partial_{x} & \rho \partial_{y} & \rho \partial_{z} & 0 \\
0 & Q & 0 & 0 & \frac{1}{o} \partial_{x} \\
0 & 0 & Q & 0 & \frac{1}{o} \partial_{y} \\
0 & 0 & 0 & Q & \frac{2}{2} \partial_{z} \\
0 & \rho c^{2} \partial_{x} & \rho c^{2} \partial_{y} & \rho c^{2} \partial_{z} & Q
\end{array}\right)\left(\begin{array}{c}
\rho \\
u \\
v \\
w \\
p
\end{array}\right)=0
$$

where $Q=\vec{u} \cdot \vec{\nabla}\left(\vec{u} \equiv\left(u \cdot v^{*}, u\right)\right.$ denotes the velocity vector), with the solid wall boundary condition

$$
\bar{u} \cdot \bar{n}=0 .
$$

Additional conditions that are given at the inflow and outflow boundaries in terms of characteristic variables are not used explicitly in the derivation of the approximate Hessian.

\subsection{The Small Disturbance Minimization Problem}

Following the same argument given in Sec.4, the small disturbance cost function is given in Eq.(4.7). The stat.e equations (5.1) are perturbed in the vicinity of the minimum and tis: perturbation variables solve the linearized Euler equations which up to low order terms are given by the same matrix operator as above. Following the localization and half space 
approximation we set the local coordinate system on the boundary such that the flow is in the $\mathrm{x}$-direction $(\vec{u}=(u, 0,0)$. The small disturbance wall boundary condition is givis by (the perturbation variables are dencted by $\dot{\rho}, \hat{\bar{u}}$, and $\dot{p}$ )

$$
-\tilde{u}=u \tilde{a}_{x}
$$

where we have omitted as before the zero order terms in $\dot{\alpha}$.

\subsection{The Adjoint Equations}

By standard variational calculus the gradient of the cost function is given by

$$
g=-u \rho\left(\lambda_{1}-c^{2} \lambda_{3}\right)_{s}-\frac{\left(p-p^{-}\right)^{2}}{R}+2 p_{n}\left(p-p^{*}\right)
$$

where $\left(\lambda_{1}, \vec{\lambda}, \lambda_{5}\right)$ is the solution of the following system of equations in $\mathbb{R}_{+}^{3}$.

$$
\begin{aligned}
\operatorname{div}\left(\lambda_{1} \vec{u}\right) & =0 \\
\operatorname{grad}\left(\rho \lambda_{1}\right)+\operatorname{grad}(\vec{u} \cdot \vec{\lambda})+c^{2} \operatorname{grad}\left(\rho \lambda_{5}\right) & =0 \\
\operatorname{div}\left(\frac{1}{\rho} \vec{\lambda}\right)+\operatorname{div}\left(\lambda_{3} \vec{u}\right) & =0 .
\end{aligned}
$$

The boundary condition on $\partial R_{+}^{3}$ is given by

$$
-\frac{1}{\rho} \lambda_{4}+2 \tilde{p}\left(p-p^{*}\right)=0 .
$$

An additional requirement is consistency with the far-field boundary conditions of the unperturbed problem; i.e., the solution is bounded for the subsonic case $(M<1)$ and that no waves propagate in the direction of $-\vec{u}\left(x_{0}\right)$ in the supersonic case $(M>1)$.

Neglecting zero order terms in Exs.(5.5-5.7) we arrive at the following quasi-linear form:

$$
\overline{\mathbf{L}}\left(\begin{array}{c}
\lambda_{1} \\
\lambda_{2} \\
\lambda_{3} \\
\lambda_{1} \\
\lambda_{3}
\end{array}\right) \equiv-\left(\begin{array}{ccccc}
Q & 0 & 0 & 0 & 0 \\
\rho \partial_{x} & Q & 0 & 0 & \rho c^{2} \partial_{x} \\
\rho \partial_{y} & 0 & Q & 0 & \rho c^{2} \partial_{y} \\
\rho \partial_{z} & 0 & 0 & Q & \rho c^{2} \partial_{z} \\
0 & \frac{2}{\rho} \partial_{x} & \frac{1}{p} \partial_{y} & \frac{1}{\rho} \partial_{z} & Q
\end{array}\right)\left(\begin{array}{c}
\lambda_{3} \\
\lambda_{2} \\
\lambda_{3} \\
\lambda_{1} \\
\lambda_{3}
\end{array}\right)=0 .
$$

\subsection{Local Mode Analysis}

Following the same procedure as in the full potential case we consider a perturbation in the design variable of the form

$$
\bar{a}(\bar{x})=\dot{\alpha}(\bar{k}) e^{i \bar{k} \cdot z}
$$

with $\vec{k}=\left(k_{1}, k_{2}\right)$ and $\vec{x}=(x, y)$. As a result the states and costates are perturbed by

$$
\begin{aligned}
& \dot{\vec{U}}(\vec{x})=\hat{\vec{l}}(\vec{k}) \epsilon^{i \vec{k} \cdot \vec{z}} e^{i k_{2}:} \\
& \vec{\Lambda}(\vec{x})=\hat{\vec{\Lambda}}(\vec{k}) \epsilon^{i \vec{k} \cdot z} e^{i k_{3}:}
\end{aligned}
$$


with $\dot{\vec{l}}=\left(\tilde{\rho}_{0}, \dot{\bar{u}}, \dot{p}\right)$ and $\vec{X}=\left(\lambda_{1}, \vec{\lambda}, \lambda_{3}\right)$. Introducing the notation

$$
\hat{Q}(\vec{k})=\vec{u} \cdot \vec{k}=u k_{2}
$$

and

$$
\hat{L}\left(\vec{k} . k_{3}\right)=i\left(\begin{array}{ccccc}
\hat{Q} & \rho \hat{k}_{1} & \rho k_{2} & \rho k_{3} & 0 \\
0 & \hat{Q} & 0 & 0 & \frac{k_{2}}{0} \\
0 & 0 & \hat{Q} & 0 & \frac{k_{2}}{0} \\
0 & 0 & 0 & \hat{Q} & \frac{k_{3}}{0} \\
0 & \rho c^{2} k_{1} & \rho c^{2} k_{2} & \rho c^{2} k_{3} & \hat{Q}
\end{array}\right)
$$

the form of the above solutions for the perturbation variables imply that

$$
\begin{aligned}
& \operatorname{det} \dot{L}\left(\vec{k}, k_{3}\right)=0 \\
& \operatorname{det} \hat{\bar{L}}\left(\vec{k}, \vec{k}_{3}\right)=0
\end{aligned}
$$

where $\hat{\bar{L}}$ is the adjoint of $\dot{L}$. The two relations result in a fifth-order polynomial equation for the wave numbers $\left(k_{1}, k_{2}, k_{3}\right)$.

$$
\begin{aligned}
& k_{1}^{3} u^{3}\left(k_{1}^{2} u^{2}-c^{2}\left(k_{1}^{2}+k_{2}^{2}+k_{3}^{2}\right)\right)=0 \\
& k_{2}^{3} u^{3}\left(k_{1}^{2} u^{2}-c^{2}\left(k_{1}^{2}+k_{2}^{2}+\bar{k}_{3}^{2}\right)\right)=0
\end{aligned}
$$

with the roots

$$
k_{3}^{2}=k_{3}^{2}=-\left(1-M^{2}\right) k_{1}^{2}-k_{2}^{2} \text { and } k_{1}=0 .
$$

where the Mach number is given by $M^{2}=\frac{u^{2}}{c^{2}}$. Note that the roots for $k_{3}$ and for $\bar{k}_{3}$ are identical to those obtained in the full potential case.

Let

$$
\begin{array}{lc}
\hat{\vec{V}}_{1}(\vec{k})= & \left(k_{1} \rho u c^{-2},-k_{1},-k_{2},-k_{3}, k_{1} \rho u\right) \\
\dot{\vec{V}}_{2}(\vec{k})= & \left(k_{1} \rho u c^{-2},-k_{1},-k_{2}, k_{3}, k_{1} \rho u\right) \\
\dot{\vec{V}}_{3}(\vec{k})= & (1,0,0,0,0) \\
\dot{\vec{V}}_{4}(\vec{k})= & (0,1,0,0,0) \\
\dot{\vec{V}}_{5}(\vec{k})= & \left(0,0 .-k_{3}, k_{2}, 0\right) \\
& \\
\dot{\Xi}_{1}(\vec{k})= & \left(0,-c^{2} \rho k_{1},-c^{2} k_{2} \rho \cdot-c^{2} \rho \bar{k}_{3}, k_{1} u\right) \\
\dot{\Xi}_{2}(\vec{k})= & \left(0 .-c^{2} \rho k_{1},-c^{2} k_{2} \rho \cdot c^{2} \rho \bar{k}_{3}, k_{1} u\right) \\
\dot{\Xi}_{3}(\vec{k})= & \left(-c^{2}, 0.0,0,1\right) \\
\dot{\bar{\Xi}}_{4}(\vec{k})= & (0,1,0,0,0) \\
\dot{\Xi}_{5}(\vec{k})= & \left(0,0,-k_{3}, k_{2}, 0\right) .
\end{array}
$$


The vector $\left.\hat{\vec{V}}_{1}(\vec{k}): \hat{\Xi}_{1}(\vec{k})\right]$ is the eigenvector of $\hat{L}\left(\vec{k}, k_{3}(\vec{k})\right)\left[\dot{L}\left(\vec{k}, \bar{k}_{3}(\vec{k})\right)\right]$ with a zero eigeavalue, the vector $\hat{\vec{V}}_{2}(\vec{k})\left[\hat{\vec{\Xi}}_{2}(\vec{k})_{j}\right.$ is the eigenvector of $\hat{L}\left(\vec{k} .-k_{3}(\vec{k})\right)\left[\hat{\bar{L}}\left(\vec{k},-\dot{k}_{3}(\vec{k})\right)\right]$ with a zero eigenvalue and the vectors $\left.\hat{\vec{V}}_{3.4,5}(\vec{k}) \mid \hat{\bar{\Xi}}_{3.4 .5}(\vec{k})\right]$ are the eigenvectors of $\hat{L}\left(0, k_{3}, k_{3}\right)\left\{\hat{\bar{L}}\left(0, k_{2}, \bar{k}_{3}\right)\right]$ with a zero eigenvalue.

The eigenvectors which correspond to $k_{1}=0,\left(\overrightarrow{\vec{V}}_{3,4,5}(\vec{k})\right.$ and $\left.\hat{\vec{\Xi}}_{3,4,5}(\vec{k})\right)$, represent waves coming from $-\infty$ to $\infty$, in the half space coordinates, narallel to the surface $\partial \mathbb{R}_{t}^{3}$ in the analysis problem, and from $\infty$ to $-\infty$ in the adjoint problem and therefore do not play a role in the analysis.

The eigenvectors which correspond to $k_{3}=k_{3}(\vec{k})$ and $\vec{k}_{3}=\bar{k}_{3}(\vec{k})$ a $\vec{a}^{-}$relating changes in the designed surface with the flow field. However, only $\hat{\vec{V}}_{1}(\vec{k})$ and $\hat{\Xi}_{1}(\vec{k})$ are consistent with the far-field boundary condjtions as discussed in Sec. 4.3 .

We look for amplitudes of the vector solutions $\hat{l}(\vec{k})$ in $(5.10)$ and $\vec{A}(\vec{k})$ in $(5.11)$ consistent with the boundary conditions. From the boundary condition of the state equation (5.3) we get (see (3.12) together with $\widetilde{V}_{1}$ in $(5.15)$ )

$$
k_{3} \dot{\beta}(\vec{k})=u \dot{i} k_{1} \hat{\alpha}(\vec{k})
$$

The boundary condition of the costate equation (5.8) (see (3.17) together with the definition of $\hat{\Xi}_{1}$ in (5.16)) implies

$$
c^{2} \bar{k}_{3} \hat{\vec{\beta}}(\vec{k})=-2 k_{1} \rho u \hat{\beta}(\vec{k})
$$

and the design equation (5.4) implies

$$
\dot{g}(\vec{k})=\rho u^{2} i k_{1}^{2} c^{2} \hat{\bar{\beta}}(\vec{k})+\text { l.o.t }
$$

Finally, substituting of $(5.17)$ in $(5.18)$ and of $(5.18)$ in $(5.19)$ yields

$$
\hat{H}(\bar{k})=-2 \rho^{2} u^{4} \frac{k_{1}^{4}}{k_{3} k_{3}}=2 \rho^{2} u^{4} \frac{k_{1}^{4}}{\left(1-M^{2}\right) k_{1}^{2}+k_{2}^{2}} .
$$

\section{Analysis of the Hessian}

In the previous sections the symbol for an approximate Hessian near the minimum was obtained, namely,

$$
\hat{H}\left(k_{1}, k_{2}\right)=2 \rho^{2} u^{4} h\left(k_{1}, k_{2}\right)
$$

with

$$
h\left(k_{1}, k_{2}\right)=\frac{k_{1}^{4}}{\mid k_{1}^{2}\left(1-M^{2}\right)+k_{2}^{2} !} .
$$

The fact that the same Hessian is obtained both for the fuil potential ey ation and for Euler equations implies that for the purpose of developing new optimization algorithms it is enough to consider the full potential equations. Although the Euler equations presents additional difficulties compared with the full potential equation for the analysis problem no additional ones exist as far as the optimization is concerned. 
The Hessian's symbol in the discrete space can be ubtained in an analog way. The result will then depend on the specific discretization we use to solve the equations. One can get $\rightarrow$ crude approximation of the discrete Hessian by replacing the wave number $k_{1}$ with the discrete wave number $\theta_{1}$ (and $k_{2}$ with $\theta_{2}$ ).

$$
\left(k_{1}, k_{2}\right)=\left(\frac{\theta_{1}}{h_{1}}, \frac{\theta_{2}}{h_{2}}\right)
$$

where $\left(h_{1}, h_{2}\right)$ are the mesh-sizes in the $(x, y)$ directions respectively.

\subsection{Two Dimensions}

In two dimensions the $x_{2}$ direction does not exist and thus in the Fourier space we set $k_{2}=0$ in Eq.(6.2) resulting in

$$
h\left(k_{1}\right)=\frac{k_{1}^{2}}{\left|1-M^{2}\right|} .
$$

Substitution of $k_{1}=\frac{\theta_{1}}{h_{1}}$ in (6.3) implies that the condition number of the Hessian 3 cales with the grid mesh-size as $O\left(\frac{1}{k^{2}}\right)$. Thus, the Hessian is ill-conditioned and its condition number increases quadratically in the discretization parameter as the grid is refined. Therefore, a good estimate of the Hessian is required to obtain a fast convergence of the optimization process.

\subsection{Three Dimensions}

In three dimensions the properties of the Hessian are deternined by

$$
h\left(k_{1}, k_{2}\right)=\frac{k_{1}^{4}}{\left|k_{1}^{2}\left(1-M^{2}\right)+k_{2}^{2}\right|} .
$$

For fixed wave number in the stream direction, $k_{1}=$ const. the symbot approaches zero as the wave number $k_{2}$ approaches infinity. This means that the cost function is nearly flat with respect to perturbations in the shape which are highly oscillatory in the direction perpendicular to the flow. This might explain recent nemerical results showing that the wing surface is likely to develop oscillations in the spanwise direction [9]. Note that the above oscillations do not appear in the $2 D$ problem which indicates that the $3 D$ aerodynamic optimal shape design is inherertly a more difficult problem than the $2 D$ problem.

\section{Regularization}

As discussed in Sec.6.2 the three dimensional problem is ill-posed and as a result oscillations are expected to appear in the direction perpendicular to that of the flow. In order to eliminate this phenomenon it is necessary either to penalize the cost function or alternatively to represent the shape as a finite sum si base functions which are smooth in the direction perpendicular to that of the flow (roughly the spanwise direction). 
In this section we propose a natural penalty of the cost function which will prevent the oscillations withou' increasing the computational cost of the preconditioner. The penaity term has the following form.

$$
F_{r}=\eta \int_{\Gamma}(T \alpha)^{2} d \sigma
$$

where $\eta$ is a positive parameter and $T$ is an operator whase symbol is

$$
\hat{T}\left(k_{1}, k_{2}\right)=\frac{k_{2}^{2}}{\left|k_{1}^{2}\left(1-M^{2}\right)+k_{2}^{2}\right|} .
$$

This results in a modified Hessian given by

$$
\hat{H}\left(k_{1}, k_{2}\right)+2 \eta \frac{k_{2}^{2}}{\mid k_{1}^{2}\left(1-M^{2}\right)+k_{2}^{2}}
$$

which reaches asymptotically a constant as $k_{2}$ increases, for fixed value of $k_{1}$. Thus, for large

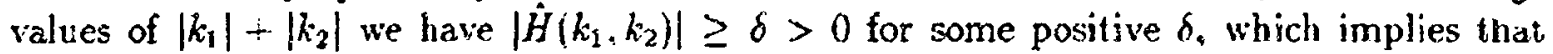
for the modified problem the high frequency perturbations in the shape are well behaved. As the shape $I$ converges to the mininum, $\eta$ can be decreased, resu'ting in a solution which is regularized in the direction perpendiculas to the flow. The implementation of the regularization is discussed in the next section.

\section{Preconditioning}

In Sec. 6 we concluded that aerodynamic optimization are ill-conditioned problems. Therefore having second order information is crucial for effective convergence. However. the explicii solution of a Vewton step. $H \bar{\alpha}=-g$, requires to comput the Hessian, $H$, explicitly and then to invert it. Tinis will become practically impossible for a realistic aerodynarnic optimization problem computed numerically on a fine mesh and having a large number of design variables. Using low rank quasj-Newton methods, such as BFGS, will deteriorate as the number of design variables increases. We therefore suggest to approximate the Newton step in the differential level (infinite dimension), using the Hessian's symbol, and then to project the result onto the finite dimensianal design space which is used in practice.

The equation defining the lewton direction, for $a$, in Fourier space is given by

$$
\hat{H}\left(k_{1}, k_{2}\right) \dot{\alpha}\left(k_{1}, k_{2}\right)=-\hat{g}\left(k_{1}, k_{2}\right)
$$

or explicitly by using the symbol $(6.1)$

$$
\left(2 \rho^{2} u^{4} k_{1}^{4}+2 \eta k_{2}^{2}\right) \dot{\alpha}\left(k_{1}, k_{2}\right)=-\left|k_{1}^{2}\left(1-\lambda \mu^{2}\right)+k_{2}^{2}\right| \hat{g}\left(k_{1}, k_{2}\right)
$$

The symbol in the right hand side of equation (8.1) corresponds to a non-local operator in the real space. The term which multiplies $\eta$ accounts for the regularization penalty term discussed in the previous section ( $\eta$ should be set to zero in two dimensional problems) 


\subsection{Preconditioner for the Small Disturbance Problem}

lsing the relation between $k_{3}, k_{1}$ and $k_{2}$,

$$
\left|k_{1}^{2}\left(1-M M^{2}\right)+{ }^{\prime 2}\right|=-\left(i k_{i}\right)\left(i \dot{k}_{3}\right)
$$

we arrive at

$$
\left(2 \rho^{2} u^{4} k_{\jmath}^{4}+2 \eta k_{2}^{2}\right) \hat{\alpha}\left(k_{1}, k_{2}\right)=\left\{i k_{3}\right)\left(i \bar{k}_{3}\right) \hat{g}\left(k_{1}, k_{2}\right)
$$

This implies the following equation in real space,

$$
2 \rho^{2} u^{4} \frac{\partial^{4} \tilde{\alpha}}{\partial x^{4}}-2 \eta \frac{\partial^{2} \bar{\alpha}}{\partial^{2} y}-\mu \hat{\alpha}=\frac{\partial \tilde{y}^{(j)}}{\partial z}+\mu g \quad \text { m } \partial K_{+}^{3} \text {. }
$$

The terms which multiply $\mu$ are added both to ensure a unique solution to Eq.(8.3) and to account for the low-frequencies. Note that Eq. (8.2) is a good approximation for the symbol of the Veuton equation only in the high-frequencies. In the low-frequencies the terms multiplying $\mu$, in Eq. (8.3), are dominant and result in a steepest descent step, while in the high-frequency regime the diminish and a Quasi-Newton step is taken. The term $t^{\text {(t) }}$ satisfies the following coupled PDE sysiem

$$
\begin{aligned}
& \left(1-M^{2}\right) w_{x z}^{(1)}-y_{y y}^{(1)}+i_{z=}^{(1)}=0 \text { on } I R_{+}^{3} \\
& i^{(1)}=\frac{\partial \dot{u}^{(2)}}{\partial z} \text { on } \partial R_{+}^{3} \\
& \left(1-M^{2}\right) \psi_{z x}^{(2)}-i_{y y}^{(2)}-i_{z z}^{i(2)}=0 \text { on } I R_{+}^{3} \\
& \dot{\psi}^{(2)}=g \text { on } \partial K_{+}^{3} \text {. }
\end{aligned}
$$

We also require that the solution be bounded and that in the supersonic regime $(M>1)$ no waves propagate in the positive $x$-direction in Eq. $(8.5)$ and that no waves propagate in the negative $\mathrm{x}$-direction in $\mathrm{Eq} .(8.7)$. Note that the operator $\mathrm{T}$ in Eq.(7.1) need not be explicitly evaluated. By adding the $\eta$ term in (8.2) and solving Eqs.(8.3-8.i) we account for such an operator. A similar preconditioner can be derived for the small disturbance Euler equations.

\subsubsection{Purely Subsonic Flow}

In a purely subsonic flow it is unrecessary to go through the above procedure since

$$
\left|k_{1}^{2}\left(1-M^{2}\right)+k_{2}^{2}\right|=k_{1}^{2}\left(1-M^{2}\right) \div k_{2}^{2} \text {. }
$$

Therefore the following PDE should be solved on I' only:

$$
2 \rho^{2} u^{4} \frac{\partial^{4} \dot{\alpha}}{\partial x^{4}}-2 \eta \frac{\partial^{2} \dot{\alpha}}{\partial^{2} y}-\mu \dot{\alpha}=\left(1-M^{2}\right) \frac{\partial^{2} g}{\partial^{2} x}+\frac{\partial^{2} g}{\partial^{2} y}+\mu g
$$




\section{\$.2 Practical Implementation}

In practice equatiois (8.4-8.7) are replaced by full potential equations defined on the domain $\Omega$ as shown in the following. Let $\phi$ be the solution of the full potential equation (4.2). We define

$$
\begin{aligned}
& \psi^{\prime}(1)=\hat{p}+\varepsilon \hat{q}^{j}(1) \\
& \psi^{\prime}(2)=\dot{p}+\varepsilon u^{j}(z)
\end{aligned}
$$

where $\varepsilon \ll 1$. We claim that the preconditioning equation for $a$ is given by

$$
2 \rho^{2}(\nabla \varphi \cdot \nabla)^{4} \dot{\alpha}-2 n(\hat{\epsilon} \cdot \nabla)^{2} \dot{\alpha}+\mu \dot{\alpha}=-\frac{1}{\varepsilon} \frac{\partial}{\partial n}\left(\psi^{(1)}-\varphi\right)+\mu g \quad \text { on } \Gamma
$$

where $\vec{e}$ is a unit vector perpendicular to $\nabla \varphi$ and to $\bar{n}$ (roughly in the spanwise direction): $\frac{\nabla_{\varphi}}{1 \nabla \bullet i} \times \vec{e}=\vec{n}$. The function $\psi^{(i)}$ is the solution of the following coupled PDE system:

$$
\begin{array}{cc}
\nabla \rho\left(\psi^{(1)}\right) \nabla \psi^{(1)}=0 & \text { on } \Omega \\
\psi^{(1)}=-\frac{\partial}{\partial n}\left(\psi^{(2)}-\varphi\right)+\varphi & \text { on } \Gamma \\
\psi^{(1)}=\varphi & \text { on } \partial \Omega-\Gamma \\
\nabla \rho\left(\psi^{(2)}\right) \nabla \psi^{(2)}=0 & \text { on } \Omega \\
\psi^{(2)}=\varepsilon g+\varphi & \text { on } \Gamma \\
\psi^{(2)}=\psi & \text { on } \partial \Omega_{0}
\end{array}
$$

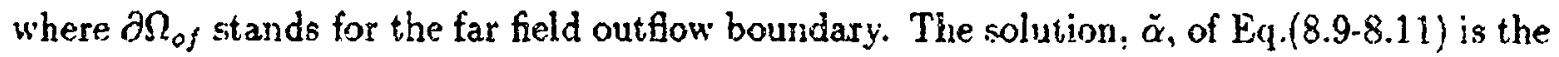
preconditioned search direction to be used in optimization algorithms. This search direction will aroid oscillations in the shape in the direction perpendicular to the flow and will require many fewer optimization steps to solve the problem. For the Euler equations an analog of Eqs.(8.10-8.11) can be derived using the corresponding small disturbance preconditioner.

\subsection{Implementation in a Finite Dimensional Design Space}

In a finite dimensional subspace we replace a in Eq.(8.9) by (see (2.15))

$$
\dot{\alpha}(\vec{x})=\sum_{j=1}^{N} \tilde{\alpha}_{j} f_{j}(\tilde{x}) .
$$

We then take the inner product of Eq. (3.9) with $f_{k}$ for $k=1 \cdots N$, resulting in a linear set of equations for $a_{j}$ :

$$
(h+\mu l) \overrightarrow{\ddot{\alpha}}=-\vec{b}+\mu \vec{y},
$$

where $I$ is the unit matrix and $\mu$ is a positive parameter. as in Eq.(8.3). $h$ is an $N \times N$ matrix in which an element $h_{1}$, is given by

$$
h_{i j}=\left\langle 2 \rho^{2}\left(\nabla_{\varphi} \cdot \nabla\right)^{4} f_{i}-2 \eta(\vec{\epsilon} \cdot \nabla)^{2} f_{i}, f_{j}\right\rangle_{L^{2}(\Gamma)}
$$


$\overrightarrow{\tilde{\alpha}}$ is the finite dimensional design variable $\overrightarrow{\tilde{\alpha}}=\left(\tilde{\alpha}_{1} \cdots, \dot{\alpha}_{N}\right)$, and an element in the right hand side vector, $\vec{b}$, is given by

$$
b_{i}=-\frac{1}{\varepsilon}\left\langle\frac{\partial}{\partial n}\left(\psi^{(i)}-\dot{r}\right) \cdot f_{i}\right\rangle_{L^{2}(\Gamma)}
$$

The solution of Eq.(8.12) is the preconditioned search direction replacing $\left(g_{1}, \cdots, g_{n}\right)$.

\section{Conclusions}

In this work new local mode analysis for cptimal shape design problems which are governed by PDEs was developed. The analysis was applied to aerodynamic shape optimization problems governed by the full potential and the Euler equations. The analysis was done in the infinite dimensional design space where arbitrary changes in the wing's shape, in the normal direction, are allowed during the course of optimization. In this case the Hessian is an infinite dimensional operator defined on a space of functions and its eigenvalue distribution served to study the well-posedness of the optimization problem as well as for deriving preconditioners to accelerate the numerical convergence of gradient based methods. In practice, however, a finite dimensional design space is commonly used for which the Hessian is a finite dimensional matrix. The application of the infinite dimensional analysis to finite dimension was obtained by a simple projection.

The analysis is local and uses freezing the coefficient to oblain a problem in half space with constant coefficients, where Fourier techniques are empioyed. The eigenvalue distribution of the Hessian is analyzed by computing its Fourier symbol. I: was shown that for two dimensional flow the Hessian is a second order differential operatoi efined on the designed boundary. In three dimension the Hessian is a pseudodifferential operaior (non-local) and its properties are much more complex. For both the full potential and Euler flow the symbols of the Hessian are identical. Therefore, the complexity of the optimization problems is the same for both, although the analysis problem for the Euler equations is more difficult.

The symbol of the Hessian implies that the three dimensional problems are ill-posed, and arbitrary oscillations in the shape can develop in the direction perpendicular to that of the flow (roughly the spanwise direction). This explains recent numerical results showing that the wing surface is highly oscillatory in the spanwise direction [9]. A regularization that involves smoothing only in that direction was introduced and analyzed. Also note that if the problem were to minimize drag. rather then matching the pressure distribution, then oscillations in the spanwise direction are not likely to appear since they will increase the surface area of the wing resulting in an increase in the drag.

The explicit form of the symbol of the Hessian also implies that these minimization problems are ill-conditioned and their condition number increases quadratically with the dimension of the design space. Therefore gradient descent method will be inefficient and second-order information, by approximating the Hessian (or its inverse). is essential for fast convergence. However, low rank quasj-Newton methods, such as BFGS, will deteriorate as the rumber of clesign variables increases. New preconditioners which approximate the inverse of the Hessian are proposed. Their numerical implementation will be presented 
elsewhere. These preconditioners are of low computational complexity for two-dimensional flow and for purely subsonic flows in three dimensions. In non-subsonic three-dimensiona! flor the preconditioning involves the solution of the full potential equation twice per each optimization step, though a substantial decrease in the number of optimization steps required to reach the minimum is anticipated. The preconditioning of the suggested regularization is straightfor wasd and requires negligible additional computational work. In case a frite dimensional design space is used the preconditioning requires the solution of a linear set of equations.

Applications of similar analysis to aeroelasijc optimization are discussed in [18?.

Acknowledgment

The anthors would like to thank R. M. Lewis for his important comments on the manuscript.

\section{References}

[1] O. Pironneau. Optimal Shape Design for Elliptic Systems, Springer Series in Computational Physics (1983).

[2] B. Mantel, J. Periaux and B. Stouflet, AGARD-FDP-VKI Special Course, Optimum Design Methods in Aerodynamics, April 25-29 (1994).

i3) A. Jameson. Aerodynamic Design Via Control Theory. Journal of Scientific Computing. 3:233-260 (1988).

[4] A. Iollo and M. Salas. Contribution to the Optimal Shape Design of Two-Dimensional Internal Flows with Emb. 3ded Shocks, ICASE Report No. 95-20 (1995).

ij; 5. Ta'asan, G. Kururila and M. D. Salas. Aerodynamic Design and Optimization in One Shot. 30th Aerospace Sciences Meeting \& Exhibit. AIA 92-0025, Jan. (1992).

[6] S. Ta asan. Trends in Aerodynamics Design and optimization: $A$. Wathematical Viewpoint. AIAA 95-1730. 12th AIAA Computational Fluid Dynamics Conference, July $19-22(1995)$.

[7] A. Dervieux, J. Malé, N. Macro, J. Périaux, B. Stoufflet and H.Q. Chen. Some Recent Advances in Optimal Shape Design For Aeronautical Flows, Proceedings of "ECCOMAS, 2nd Computational Fluid Dynamics Conference", Sep. 5-8 (1994).

[8] P. D. Frank and G. R. Shubin. A comparison of optimization-based approaches for a model computational aetodynamics design problem. J. Comput. Phys. 98. 74 (1992).

[9] W. H. Jou, W. P. Huffman, D. P. Young, R. G. Melvin, M. B. Bieterman, C. L. Hilmes and F. T. Johnson (The Boeing Company). Practical Considerations in Aerodynarnic Design Optimization. AIAA 95-1730, 12th AIAA Computational Fluid Dynamics Conference, July 19.22 (1995).

[10 .). Hadamard. Lessons on the Calculus of Variation (in Prench). Gauthier-Villards, Paris (1910). 
i1: M. J. Lighthill. A new metsud of two dimensional aesodynamic design. $R$ \& . M 1111 , Aeronautical Research Council (1945).

12] P. E. Gill. W. Murray and M. H. Wright. Practical Optimization. Academic Press (1981).

[13] E. Arian. Multigrid Methods for Optimal Shape Design Governed by Elliptic Systerns. Ply.D. Thesis, The Weizrnann Institute of Science, Israel (1994).

[14. E. Arian and S. Ta asan. Multigrid One Shot Methods for Optimal Design Problems: Infinite-Dimensional Control, YCASE Report No. 94-52 (1994).

[15: E. Arian and S. Ta asan. Shape Optimization in One Shot, Optimal Design and Control, Edited by J. Boggaard, J. Burkardt, M. Gunzburger and J. Peterson, Birkhäuser Boston Inc. (199.5).

[16] E. Arian and S. Ta asan. Smoothers for Optimization Problems, Proceedings of the Seventh Copper Mountain Conference on Multigrid Methods. April 2-7 (1995).

[17: C. Hirsch. Numerical Computation of Internal and External Flows, Vol. 2, John Wiley \& Sons (1988).

[18; E. Arian. Analysis of the Hessian for Aeroelastic Optimization. ICASE Report No. $95-84(1995)$. 


\begin{tabular}{|c|c|c|c|}
\hline \multicolumn{3}{|c|}{ REPORT DOCUMENTATION PAGE } & $\begin{array}{l}\text { Fam Aparoved } \\
\text { OAsB No. Oros-018s }\end{array}$ \\
\hline \multicolumn{4}{|c|}{ 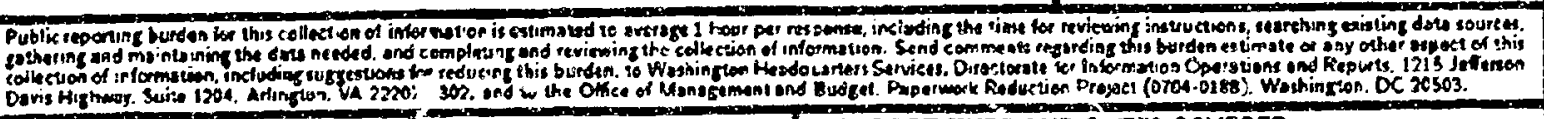 } \\
\hline 1. AGENCY USE OHCY(Lesue Slank) & $\begin{array}{l}\text { 2. REPORT OATE } \\
\text { APRI } 1996\end{array}$ & \multicolumn{2}{|c|}{$\begin{array}{l}\text { 3. REPORT TYPE AND ORTES COVERED } \\
\text { Contractor Report }\end{array}$} \\
\hline \multicolumn{3}{|c|}{$\begin{array}{l}\text { 4. TITLE AND SUBTITLE } \\
\text { ANALYSIS OE THE HESSIAN FOR AERODYNAMIC } \\
\text { OPTIMIZATION: NYISCID FLOW }\end{array}$} & \multirow[t]{2}{*}{$\begin{array}{l}\text { 5. FUMDING MUMEERS } \\
\text { C NAS1-19480 } \\
\text { WL } 505-90-52-01\end{array}$} \\
\hline \multicolumn{3}{|l|}{$\begin{array}{l}\text { 6. Aurior(S) } \\
\text { Eyal Arian } \\
\text { Shlomo Tadasan }\end{array}$} & \\
\hline \multicolumn{3}{|c|}{$\begin{array}{l}\text { 7. PERFORMING ORGAKIZATION KAME(S) ANO AOORESS(ES) } \\
\text { Institute for COMputer Applications in Science and Engineering } \\
\text { Mail Stop 132C, NASA Langley Research Center } \\
\text { Hamplon. VA } 23681-0001\end{array}$} & $\begin{array}{l}\text { 8. PEÁFORAMING ORGANIZATIOH } \\
\text { REPORT MUMBER } \\
\text { ICASE Report No. } 96-28\end{array}$ \\
\hline \multicolumn{2}{|c|}{$\begin{array}{l}\text { 9. SPOASORSAG/MONTTORING AGENCY WAME(S) AND AODRESS(ES } \\
\text { National Aerontutics and Space Administration } \\
\text { Langley Research Center } \\
\text { Hampton, VA 23681-0001 }\end{array}$} & $\begin{array}{l}\text { 10. SPOWS: } \\
\text { AGEMC } \\
\text { NASA } \\
\text { ICASE: }\end{array}$ & $\begin{array}{l}\text { 10. SPONSORING/WOHITORING } \\
\text { AGENCY REPOKT WUABER } \\
\text { NASA CR-198328 } \\
\text { ICASE; REPCIR NO. } 96-28\end{array}$ \\
\hline \multicolumn{4}{|c|}{$\begin{array}{l}\text { i1. Supptementany Notes } \\
\text { Langley Technical Monitor: Dennis M. Bushrell } \\
\text { Final Report } \\
\text { To be sabmilted to the Journal of Computational Physics. }\end{array}$} \\
\hline \multicolumn{2}{|c|}{$\begin{array}{l}\text { 12. DISTRIBUTIDN/AVAILABHLITY STATEMENT } \\
\text { Cnclatgified-Unimited } \\
\text { Subject Category } 64\end{array}$} & \multicolumn{2}{|c|}{ 12b. DISTAIQUTIOAN CODE } \\
\hline \multicolumn{4}{|c|}{ 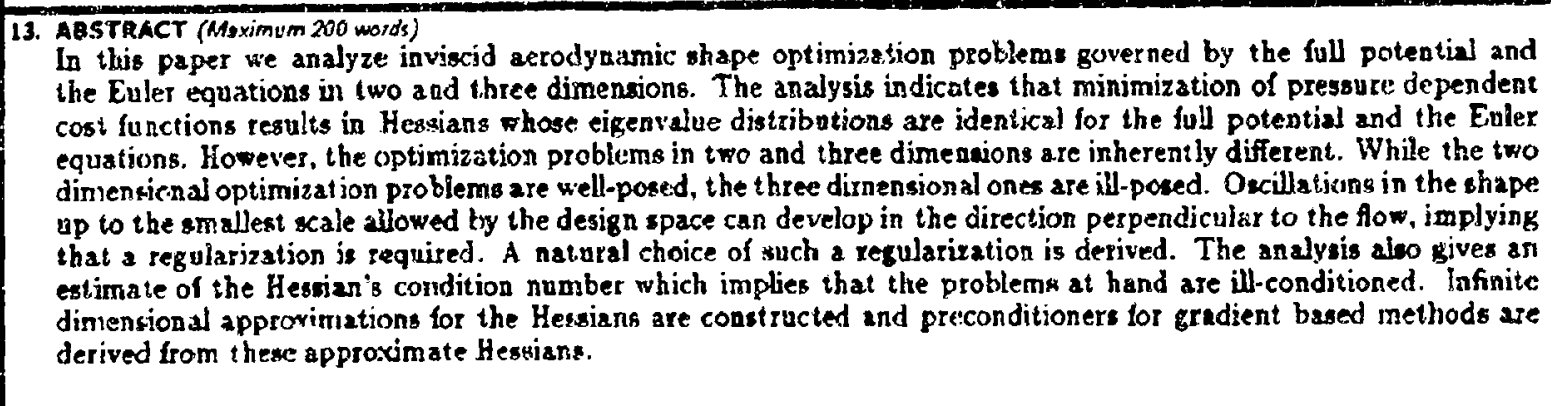 } \\
\hline \multirow{2}{*}{\multicolumn{3}{|c|}{$\begin{array}{l}\text { 14. SUPJECT TERMS } \\
\text { Aerodynamic Optimization; Optimal Shape; Full Potential Eguations; Enler Fquations: } \\
\text { Heasian; Preconditioning; Regularization }\end{array}$}} & $\begin{array}{l}\text { 15. NUMBER OF PAGES } \\
22\end{array}$ \\
\hline & & & $\begin{array}{l}\text { 16. PRLCE CODE } \\
\text { AO3 }\end{array}$ \\
\hline $\begin{array}{l}\text { 17. SECURITY CLASBIf ICAYION } \\
\text { OF REP ORT } \\
\text { Unclassified }\end{array}$ & $\begin{array}{l}\text { 16. SECURITY CLABSIFICATION } \\
\text { Of YHIS PAGE } \\
\text { Inclasuified }\end{array}$ & $\begin{array}{l}\text { 39. SECURITY CLASSIFICATION } \\
\text { OF AOSTRACT }\end{array}$ & $\begin{array}{l}\text { 20. LIMITATION } \\
\text { OF ADSTAACT }\end{array}$ \\
\hline 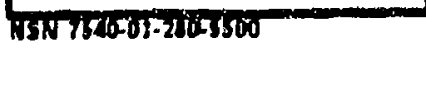 & & & 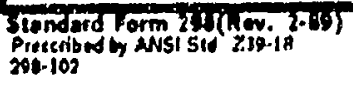 \\
\hline
\end{tabular}

